\title{
Dynamic stabilization of metal oxide-water interfaces
}

\author{
Martin E. McBriarty, ${ }^{*} \dagger$ Guido F. von Rudorff, ${ }^{\dagger}$ Joanne E. Stubbs, ${ }^{\S}$ Peter J. Eng, ${ }^{\S}$ Jochen Blumberger, ${ }^{\ddagger}$ \\ Kevin M. Rosso* ${ }^{* \dagger}$
}

†Physical Sciences Division, Pacific Northwest National Laboratory, Richland, Washington 99352, U.S.A.

tDepartment of Physics and Astronomy, University College London, London WC1E 6BT, U.K.

${ }^{\S}$ Center for Advanced Radiation Sources, University of Chicago, Chicago, Illinois 60439, U.S.A.

Supporting Information Placeholder

\begin{abstract}
The interaction of water with metal oxide surfaces plays a crucial role in the catalytic and geochemical behavior of metal oxides. In a vast majority of studies, the interfacial structure is assumed to arise from a relatively static lowest energy configuration of atoms, even at room temperature. Using hematite $(\alpha-$ $\mathrm{Fe}_{2} \mathrm{O}_{3}$ ) as a model oxide, we show through a direct comparison of in situ synchrotron X-ray scattering with density functional theorybased molecular dynamics (DFT-MD) simulations that the struc-

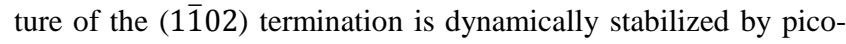
second water exchange. Simulations show frequent exchanges between terminal aquo groups and adsorbed water in locations and with partial residence times consistent with experimentally determined atomic sites and fractional occupancies. Frequent water exchange occurs even for an ultrathin adsorbed water film persisting on the surface under a dry atmosphere. The resulting time-averaged interfacial structure consists of a ridged lateral arrangement of adsorbed water molecules hydrogen bonded to terminal aquo groups. Surface $\mathrm{p} K_{\mathrm{a}}$ prediction based on bond valence analysis suggests that water exchange will influence the proton transfer reactions underlying the acid/base reactivity at the interface. Our findings provide important new insights for understanding complex interfacial chemical processes at metal oxide-water interfaces.
\end{abstract}

The interfaces between metal oxides and water are among the most important in nature and in emerging energy applications, with wide ranging impacts from photocatalytic water splitting ${ }^{1-4}$ to the geochemical cycling of elements ${ }^{5,6}$. Key chemical processes such as adsorption, electron transfer, growth, and dissolution all depend principally on the atomic structure adopted at these interfaces. For example, dissolution and solute adsorption are regulated by the structure of interfacial water ${ }^{7,8}$. Surface acid/base chemistry ${ }^{9,10}$ arises from the types and arrangement of terminal metal-coordinating aquo/hydroxyl groups ${ }^{3,11-13}$.

At room temperature an interface is at dynamic equilibrium. In principle, the average interfacial structure depends on the interplay of relatively static atoms at the solid surface with relatively dynamic overlying water molecules. Simulations suggest that movement of overlying water molecules can play an essential role in stabilizing the interface and influencing its chemical behavior ${ }^{14,15}$. However, simulated ${ }^{14-17}$ or spectroscopically probed ${ }^{18}$ dynamics are seldom integrated with experimentally derived interface structure models to achieve comprehensive insight into interfacial structure ${ }^{4}$. To understand and predict chemical processes at dynamically active metal oxide-water interfaces, structure and dynamics must be considered as a unified whole.
Accurate measurements of interface structure and water ordering rely on interface-sensitive synchrotron X-ray scattering methods ${ }^{19}$. The analysis of multiple crystal truncation rods (CTRs) provides a complete 3 -dimensional interface model ${ }^{20,21}$, but this structure is averaged over seconds to hours. Disorder parameters measured using time-averaged methods provide limited insight into interface dynamics $^{22}$. However, rigorous ab initio modeling can reveal dynamical behavior underlying time-averaged data which may not be interpretable using static structural models ${ }^{23}$. Sub-picosecond phenomena at metal oxide-water interfaces, including ligand exchange and proton dynamics, are within reach of accurate density functional theory-based molecular dynamics (DFT-MD) ${ }^{4,15-17}$.

Here we exploited a combination of CTR measurements with large-scale hybrid functional DFT-MD calculations to derive a dynamical structure model of an archetypical metal oxide interface with water. Hematite $\left(\alpha-\mathrm{Fe}_{2} \mathrm{O}_{3}\right)$ is both a naturally abundant mineral and a photoactive semiconductor of interest for heterogeneous catalysis $^{24}$. The (1102) (" $r$-cut") surface is a prominent low-index face $^{25,26}$ that exposes high electron mobility pathways in the hematite structure ${ }^{27,28}$ and reactive iron-oxo surface functional groups $^{20,29}$. Early studies suggest that the most stable termination at room temperature is an iron-deficient surface with hydroxyls between ridges of terminal aquo groups ${ }^{20,30}$, but the water structure above this surface is debated. Classical MD ${ }^{31}$ and static DFT ${ }^{30}$ calculations suggest that the first water layer localizes between the ridges of aquo groups, stabilized by hydrogen bonding. However, experimental evidence indicates that the first layer of water adsorbs more closely to terminal aquo groups, leaving void spaces above the hydroxyls ${ }^{20}$.

We performed in situ 3-dimensional CTR measurements on the hematite (1102) surface under dry flowing helium and immersed in an anoxic dilute electrolyte solution ( $5 \mathrm{mM} \mathrm{Na}_{2} \mathrm{SO}_{4}$, $\mathrm{pH}$ 7.4; see SI for solution details). CTR analysis was complemented by DFTMD simulations of the fully hydrated interface in dynamic equilibrium with an overlaying bulk water phase. The water dynamics from the simulations were essential to resolve non-intuitive details that emerge from the time-averaged structure measurements, providing robust evidence of transiently occupied surface-bound aquo ligand and adsorbed water sites undergoing frequent dynamic exchange. With strong synergy between experiment and high-level theory, the model presented here provides the most detailed and internally consistent depictions of a hematite-water interface ever presented.

Methodological details are provided in the Supporting Information (SI) and briefly summarized here. Experiments were performed on a (1102)-terminated hematite single crystal which was pre-characterized using atomic force microscopy (AFM), X-ray 
photoelectron spectroscopy (XPS) ${ }^{32-34}$, and low-energy electron diffraction (LEED) ${ }^{35}$ (Figures S1-S2). CTRs were analyzed by refining the atomic positions, fractional occupancies (relative to the (1102) layer occupancy of 7.3 atoms $\cdot \mathrm{nm}^{-2}$ ), and anisotropic disorder parameters of selected $\mathrm{Fe}$ and $\mathrm{O}$ atoms using the kinematical $\mathrm{X}$-ray scattering formalism ${ }^{36-38}$. Our experimental structure models use an orthorhombic surface cell with lattice parameters $a=5.038$ $\AA$, $b=5.434 \AA$, and $c=7.3707 \AA$, with a lattice offset parameter of 0.14 in the $b$ direction ${ }^{39,40}$. DFT-MD calculations were performed using the HSE06 hybrid functional ${ }^{41}$ with $12 \%$ Hartree-Fock exchange $^{16,17,42}$ and Grimme D3 dispersion correction ${ }^{43}$ as implemented in CP2K software ${ }^{44,45}$. Prior to DFT-MD computation, solvation structures were initialized using a classical force field $\operatorname{model}^{46,47}$.

Structures corresponding to the best fits to our CTR data (Figure S3) are shown in Figure 1, and interlayer spacings are tabulated in Table 1. Further structural details and fit parameters are listed in Tables S2 and S3 in the SI. (H atoms are not included in our CTR model; the role of protons is discussed below.) The hematite surface structures are nearly identical between wet and dry conditions, and both agree well with previous measurements ${ }^{20}$. The presence of ordered water under dry conditions is not surprising, given that adsorbed water persists at room temperature even under ultra-high vacuum (see Figure S2 and Ref. ${ }^{35}$ ). There is a significant lateral shift in the position of ${ }^{2{ }^{2}} \mathrm{O}$ between the two fits; however, ${ }^{2{ }^{2}} \mathrm{O}$ is very weakly ordered in the dry condition as indicated by its large lateral disorder $\left(0.5 \AA^{2}\right.$, see Table S3). Differences in the ${ }^{1 \mathrm{~W}} \mathrm{O}$ structure between wet and dry conditions are more subtle. Structural details agree well between our CTR measurements and DFT-MD calculations; slight discrepancies in two $\mathrm{Fe}-\mathrm{O}$ distances $\left({ }^{2} \mathrm{O}-{ }^{2} \mathrm{Fe}\right.$ and ${ }^{5} \mathrm{O}-{ }^{4} \mathrm{Fe}$ ) are related to finite slab size effects discussed in the SI.

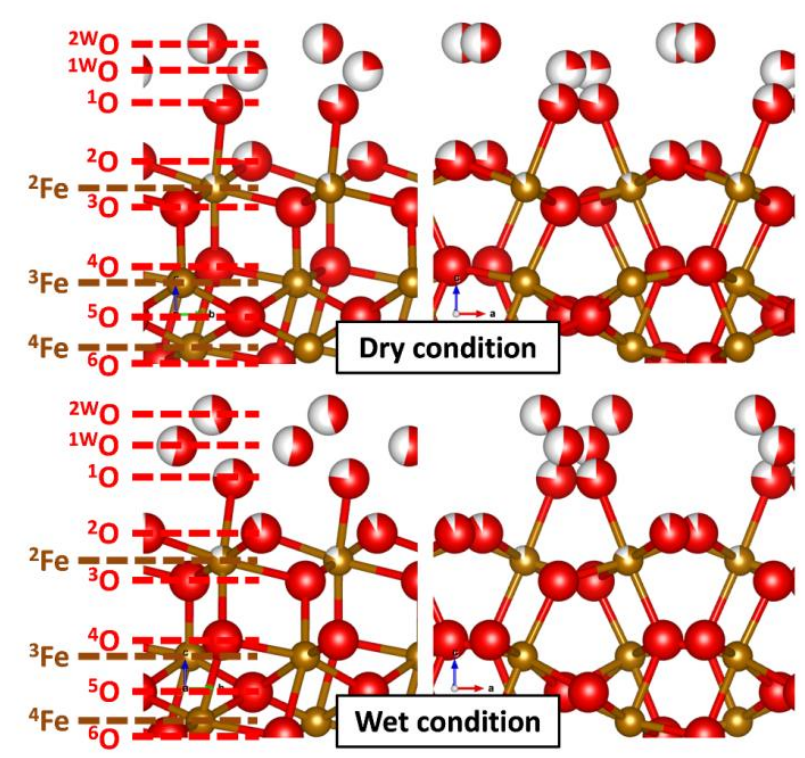

Figure 1. Ball-and-stick time-averaged models of the $r$-cut hematite-water interface derived from CTR measurements under dry (top) and wet (bottom) conditions. O atoms are shown in red and Fe in gold; the colored region of each atom indicates its fractional occupancy. Views are along the $a$ (left) and $b$ (right) directions of the orthorhombic surface cell.

While hematite lattice atoms occupy stable positions in DFTMD simulations, dynamic exchange occurs between ${ }^{1} \mathrm{O}$ and ${ }^{1,2 \mathrm{~W}} \mathrm{O}$. Time-averaged $\mathrm{O}$ density distributions in the surface normal direction are shown in Figure 2A. Distances are calibrated to the average ${ }^{2} \mathrm{O}$ position, and peak areas are normalized such that the integrated area gives the fractional $\mathrm{O}$ occupancy. The broad distribution in the DFT-MD O positions is attributed to frequent exchanges between terminal aquo groups and adsorbed water. During 19 ps of simulated surface dynamics, we observe 21 exchange events where an oxygen atom migrates between the ${ }^{1} \mathrm{O}$ and ${ }^{1 \mathrm{~W}} \mathrm{O}$ configurations. A single switch from a ${ }^{1} \mathrm{O}$ to a ${ }^{1 \mathrm{~W}} \mathrm{O}$ site typically happens in $0.2-0.3$ ps and can be stable for about 1 ps (see Figure S5). While in most instances the same aquo ligand detaches and re-binds, we also observed true exchange events in which an aquo ligand diffuses into the ${ }^{2 \mathrm{~W}} \mathrm{O}$ layer while the empty coordination site is filled by a water molecule from the ${ }^{1 \mathrm{~W}} \mathrm{O}$ layer. The model used in CTR analysis, which assumes that atoms occupy a Gaussian distribution of positions about fixed sites, cannot precisely accommodate the dynamic details of ligand exchange; this could explain discrepancies between measured and calculated partial occupancies in Figure 2A and Table S3. However, the CTR model does place the ${ }^{1 \mathrm{~W}} \mathrm{O}$ layer in the center of a region of high $\mathrm{O}$ density shown by DFT-MD, and the second CTR-derived water layer matches a lobe in the DFTMD O distribution. Considering that the only a priori chemical input to the CTR fitting is the bulk hematite crystal structure, the CTR and DFT-MD results agree exceptionally well.

Table 1. Interlayer Spacings (in ̊) for the $r$-cut HematiteWater Interface from This Work and the Literature.

\begin{tabular}{|c|c|c|c|c|}
\hline $\begin{array}{l}\text { Layer } \\
\text { Spacing }\end{array}$ & CTR (Dry) & CTR (Wet) & $\begin{array}{l}\text { DFT-MD } \\
\text { (Wet) }\end{array}$ & Humid $^{20}$ \\
\hline${ }^{2 \mathrm{~W}} \mathrm{O}-{ }^{1 \mathrm{~W}} \mathrm{O}$ & $0.6(3)^{a}$ & $0.7(2)$ & \multirow{2}{*}{ Continuous } & $0.7(1)$ \\
\hline${ }^{1 \mathrm{~W}} \mathrm{O}-{ }^{1} \mathrm{O}$ & $0.8(2)$ & $0.8(1)$ & & $1.19(7)$ \\
\hline${ }^{1} \mathrm{O}-{ }^{2} \mathrm{O}$ & $1.34(6)$ & $1.28(7)$ & 1.260 & $1.25(6)$ \\
\hline${ }^{2} \mathrm{O}-{ }^{2} \mathrm{Fe}$ & $0.63(4)$ & $0.64(4)$ & 0.934 & $0.69(4)$ \\
\hline${ }^{2} \mathrm{Fe}-{ }^{3} \mathrm{O}$ & $0.45(3)$ & $0.48(4)$ & 0.436 & $0.38(3)$ \\
\hline${ }^{3} \mathrm{O}-{ }^{4} \mathrm{O}$ & $1.40(5)$ & $1.39(7)$ & 1.410 & $1.41(4)$ \\
\hline${ }^{4} \mathrm{O}-{ }^{3} \mathrm{Fe}$ & $0.38(3)$ & $0.38(4)$ & 0.406 & $0.37(3)$ \\
\hline${ }^{3} \mathrm{Fe}-{ }^{5} \mathrm{O}$ & $0.80(3)$ & $0.84(4)$ & 0.935 & $0.77(3)$ \\
\hline${ }^{5} \mathrm{O}-{ }^{4} \mathrm{Fe}$ & $0.74(2)$ & $0.69(3)$ & 0.928 & $0.77(2)$ \\
\hline${ }^{4} \mathrm{Fe}-{ }^{6} \mathrm{O}$ & 0.354 & 0.354 & 0.375 & 0.354 \\
\hline
\end{tabular}

${ }^{a}$ Numbers in parentheses indicate the error on the last digit.

Figure 2B shows a contour map of DFT-MD-calculated O density within a $0.2 \AA$ thick plane sliced parallel to the hematite surface at a height halfway between the average positions of ${ }^{1} \mathrm{O}$ and ${ }^{1 \mathrm{~W}} \mathrm{O}$. DFT-MD again shows remarkable agreement with the experimentally measured interface structure, with the regions of highest $\mathrm{O}$ density concentrated between the ${ }^{1} \mathrm{O}$ and ${ }^{1{ }^{1}} \mathrm{O}$ sites of the wet model. The lateral ${ }^{1 \mathrm{~W}} \mathrm{O}$ positions measured under humid conditions by Tanwar et al. ${ }^{20}$ are significantly displaced from the positions in our CTR and DFT-MD models. In our model for the dry condition (Figure 2C), the partially occupied ${ }^{1 \mathrm{~W}} \mathrm{O}$ and ${ }^{1} \mathrm{O}$ sites are stacked nearly on top of each other, with only $1.00 \AA$ separating the two sites. Considering that the occupancies of these sites $\left(78 \%\right.$ for ${ }^{1} \mathrm{O}$ and $23 \%$ for ${ }^{1 \mathrm{~W}} \mathrm{O}$ ) add up to roughly $100 \%$, we conclude that dynamic exchanges of terminal aquo groups with water are occurring, even under dry conditions. In the wet condition (Figure 2D), ${ }^{1 W_{O}}$ atoms adsorb between ${ }^{1} \mathrm{O}$ sites, forming a chain with 1.61 and 1.70 $\AA \mathrm{O}-\mathrm{O}$ interaction distances. The ${ }^{1} \mathrm{O}$ occupancy is $\sim 80 \%$ and the ${ }^{1 \mathrm{~W}} \mathrm{O}$ occupancy is $\sim 50 \%$, implying the coexistence of a time-averaged stable population of water molecules at ${ }^{1} \mathrm{O}$ sites and dynamic exchange between ${ }^{1{ }^{\mathrm{W}}} \mathrm{O}$ and ${ }^{1} \mathrm{O}$ sites. The close proximity and nearly complementary occupancies of ${ }^{1 \mathrm{~W}} \mathrm{O}$ and ${ }^{2 \mathrm{~W}} \mathrm{O}$ in the wet case suggest frequent exchange occurs between these two sites as well. 
Our dynamic interface model contradicts classical MD simulations of this interface (which are based on non-dissociable molecular water models) but is consistent with a number of experimentally based assertions. In one MD study it was found that the first water layer adsorbed directly onto the ${ }^{2} \mathrm{O}$ hydroxyl site, while the second water layer formed a diffuse chain above ${ }^{1} \mathrm{O}$ aquo sites ${ }^{31}$. However, 3-dimensional CTR analyses reported here and by Tanwar et al. ${ }^{20}$ all show that ordered water molecules do not closely coordinate ${ }^{2} \mathrm{O}$ sites. These experimental observations agree with Chatman et al. ${ }^{29}$ who suggest that ${ }^{1} \mathrm{O}$ aquo ligands would screen ${ }^{2} \mathrm{O}$ sites, inhibiting the exchange of protons or ligands from ${ }^{2} \mathrm{O}$. Our laterally ordered water model also contradicts MD calculations for (1102)-terminated hematite nanoparticles which concluded that water is mobile parallel to the surface but exchange perpendicular to crystal facets is limited ${ }^{14}$. Instead, our results support Catalano et al.'s hypothesis that dynamic exchange of water might be responsible for weaker water ordering observed at the (1102) surface of $\alpha-\mathrm{Fe}_{2} \mathrm{O}_{3}$ as compared to $\alpha-\mathrm{Al}_{2} \mathrm{O}_{3}{ }^{22}$. Indeed, the simulated distribution of water at the hematite surface is much broader than for $\mathrm{TiO}_{2}$ (110), which exchanges water with solution much less frequently ${ }^{4}$. We conclude that high-level DFT-MD simulations are necessary to capture the behavior of metal oxide-water interfaces, particularly those with weakly bound water.
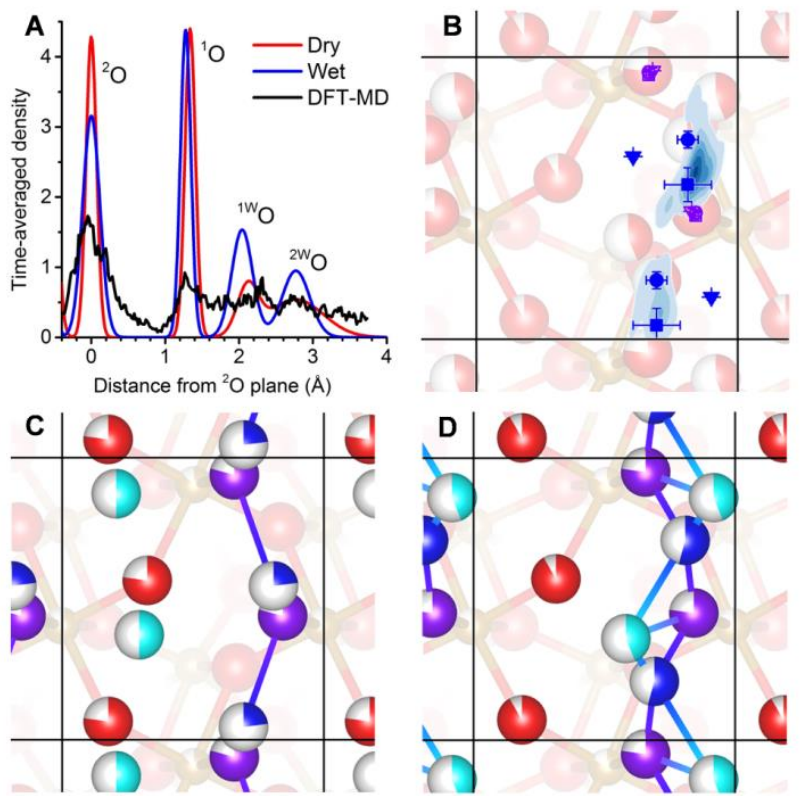

Figure 2. (A) O density along the surface normal direction derived from CTR fits (total electron density) and DFT-MD calculations (O density only). (B) Contour map of time-averaged $\mathrm{O}$ density halfway between ${ }^{1 \mathrm{~W}} \mathrm{O}$ and ${ }^{1} \mathrm{O}$ layers in the DFT-MD model relative to the surface structure of the wet CTR model. Darker regions indicate higher $\mathrm{O}$ density. Experimental positions of ${ }^{1} \mathrm{O}$ (purple open symbols) and ${ }^{1 w_{O}}$ (blue filled symbols) are overlaid for the dry (squares), wet (circles), and humid ${ }^{20}$ (triangles) conditions. (C,D) Views along the surface normal direction of the (C) dry and (D) wet CTR models. Highlighted atoms show ${ }^{2} \mathrm{O}$ (red), ${ }^{1} \mathrm{O}$ (purple), ${ }^{1 \mathrm{~W}} \mathrm{O}$ (blue), and ${ }^{2 \mathrm{~W}} \mathrm{O}$ (cyan) species, and lines are drawn between atoms with interaction distances of $2.5 \AA$ or less.

Our findings can be extended to address the relationship between structure, dynamics, and acid/base reactivity. CTR models only account for oxygen and iron atoms because of the weak X-ray scattering contribution of hydrogen. However, hydrogen distribution can be inferred based on chemical assumptions. Table $\mathrm{S} 4$ lists bond valence sums (BVS $)^{48}$ for all $\mathrm{Fe}-\mathrm{O}$ interactions near the hematite- water interface. Assuming that protonation accounts for the difference between BVS and the expected $\mathrm{O}$ valence of 2 , our static CTR models suggest that ${ }^{1} \mathrm{O}$ is normally doubly protonated, ${ }^{2} \mathrm{O}$ is consistently singly protonated, and ${ }^{3} \mathrm{O}$ is normally not protonated. To test the likelihood of different protonation states, DFT-MD calculations were initialized with either ${ }^{1} \mathrm{O},{ }^{2} \mathrm{O}$, and ${ }^{3} \mathrm{O}$ all singly protonated, or ${ }^{1} \mathrm{O}$ doubly protonated, ${ }^{2} \mathrm{O}$ singly protonated, and ${ }^{3} \mathrm{O}$ not protonated. While the second protonation configuration is stable over several picoseconds for all initial solvation configurations, the first protonation configuration quickly relaxes into the second one. In particular, for the second configuration, no proton migrates to a ${ }^{3} \mathrm{O}$ site. This suggests a stable configuration that is dominated by a termination with ${ }^{1} \mathrm{O}$ doubly protonated and ${ }^{2} \mathrm{O}$ singly protonated, in line with bond valence expectations.

Tanwar et al..$^{20}$ calculated a static hydration and protonation model using DFT and analyzed it with the method of Hiemstra et $a l .{ }^{9}$, concluding that ${ }^{1} \mathrm{OH}_{2}$ species have labile protons at circumneutral $\mathrm{pH}\left(\mathrm{p} K_{\mathrm{a}} \approx 2\right)$, while ${ }^{2} \mathrm{OH}$ species remain mostly protonated $\left(\mathrm{p} K_{\mathrm{a}} \approx 8\right)$. We measure similar interface structures and $\mathrm{BVS}$ for ${ }^{1} \mathrm{O}$ and ${ }^{2} \mathrm{O}$, but in the absence of a static protonation model we estimate $\mathrm{p} K_{\mathrm{a}}$ values using the more general method of Bickmore et al ${ }^{10}$, detailed in the SI. We calculate a $\mathrm{p} K_{\mathrm{a}}$ of 7.6 (9.1) for ${ }^{1} \mathrm{O}$ and 17.5 (17.2) for ${ }^{2} \mathrm{O}$ in the wet (dry) condition, and applying the same method to Tanwar et al.'s measured interface structure ${ }^{20}$ yields a $\mathrm{p} K_{\mathrm{a}}$ of 10.9 for ${ }^{1} \mathrm{O}$ and 12.4 for ${ }^{2} \mathrm{O}$. The ${ }^{1} \mathrm{O}$ values may be compared to the predicted $\mathrm{p} K_{\mathrm{a}}$ of 5.34 for the first deprotonation of a hexaaquo $\mathrm{Fe}^{3+}$ cation ${ }^{10}$. The higher $\mathrm{p} K$ a values calculated using Bickmore et al.'s method suggest that protons at the $r$-cut hematite surface may not be as labile as previously thought. In fact, the circumneutral $\mathrm{p} K \mathrm{a}$ for ${ }^{1} \mathrm{O}$ in the wet condition means that proton and aquo ligand exchange could occur concurrently. However, substantial disagreement between $\mathrm{p} K_{\text {a }}$ calculation methods motivates an ongoing need for a more refined theory of the acid-base behavior of metal oxide surfaces ${ }^{15,49}$ which would ideally account for ligand dynamics. The relationship between proton behavior and dynamic exchange of aquo groups with water will be discussed in future work.

In this Communication, we demonstrate for the first time that frequent water exchange defines the equilibrium structural state of a metal oxide-water interface at room temperature. Time-averaged structure models must be interpreted in light of highly accurate time-resolved simulations to fully understand the behavior of dynamically active surfaces in solution. The next step is to develop the linkages between the dynamical stabilization of the interface and important chemical phenomena such as surface acidity or electron transfer. Considering the increasing availability of high-quality theoretical and experimental methods including ultrafast spectroscopy, further studies establishing these linkages are now within reach, including assessing the roles of $\mathrm{pH}$, solute type, ionic strength, and applied electrochemical potential. This approach promises better predictive models for the interfacial phenomena that govern dissolution, adsorption, and electron transfer at metal oxide-water interfaces.

\section{ASSOCIATED CONTENT}

\section{Supporting Information}

The Supporting Information is available free of charge on the ACS Publications website.

Experimental and computational methods; characterization of the $r$-cut hematite surface using AFM, XPS, and LEED; CTR data, best fits, and fit parameters; water dynamics from DFT-MD; BVS data and discussion (PDF)

\section{AUTHOR INFORMATION}

\section{Corresponding Author}


*E-mail: kevin.rosso@pnnl.gov (K.M.R.)

*E-mail: martin.mcbriarty@pnnl.gov (M.E.M.)

\section{Notes}

The authors declare no competing financial interests.

\section{ACKNOWLEDGMENT}

This material is based upon work supported by the U.S. Department of Energy (DOE), Office of Science, Office of Basic Energy Sciences (BES), Chemical Sciences, Geosciences, and Biosciences Division through its Geosciences program at Pacific Northwest National Laboratory (PNNL). PNNL is a multiprogram national laboratory operated by Battelle Memorial Institute under Contract No. DE-AC05-76RL01830 for the U.S. DOE. The research was performed in part using the Cascade supercomputer and experimental capabilities at the Environmental and Molecular Sciences Laboratory (EMSL), a DOE Office of Science User Facility sponsored by the Office of Biological and Environmental Research. CTR measurements were performed at GeoSoilEnviroCARS (The University of Chicago, Sector 13), Advanced Photon Source (APS), Argonne National Laboratory. GeoSoilEnviroCARS is supported by the National Science Foundation - Earth Sciences (EAR-1128799) and DOE - Geosciences (DE-FG02-94ER14466). The APS is a U.S. DOE Office of Science User Facility operated by Argonne National Laboratory under Contract No. DE-AC02-06CH11357. G.v.R. gratefully acknowledges a PhD studentship co-sponsored by University College London and PNNL through its BES Geosciences program. MD simulations were carried out on ARCHER, the UK national HPC facility (Edinburgh), to which access was granted via the ARCHER Leadership pilot call and the Materials Chemistry Consortium (EPSRC grant EP/L000202). Data analysis was carried out with computing resources provided through a Microsoft Azure Sponsorship and Amazon AWS Research Grant and supported by software made available by Tableau Inc. We thank Tim Droubay for assistance with LEED and XPS measurements.

\section{REFERENCES}

(1) Sivula, K.; Le Formal, F.; Grätzel, M. ChemSusChem 2011, 4, 432.

(2) Chatman, S.; Zarzycki, P.; Rosso, K. M. ACS Appl. Mater. Interfaces 2015, 7, 1550.

(3) Plaza, M.; Huang, X.; Ko, J. Y. P.; Shen, M.; Simpson, B. H.; Rodríguez-López, J.; Ritzert, N. L.; Letchworth-Weaver, K.; Gunceler, D.; Schlom, D. G.; Arias, T. A.; Brock, J. D.; Abruña, H. D. J. Am. Chem. Soc. 2016, 138, 7816.

(4) Hussain, H.; Tocci, G.; Woolcot, T.; Torrelles, X.; Pang, C. L.; Humphrey, D. S.; Yim, C. M.; Grinter, D. C.; Cabailh, G.; Bikondoa, O.; Lindsay, R.; Zegenhagen, J.; Michaelides, A.; Thornton, G. Nat Mater 2016, doi:10.1038/nmat 4793 . 3233.

(5) Stumm, W.; Sulzberger, B. Geochim. Cosmochim. Acta 1992, 56,

(6) Neal, A. L.; Rosso, K. M.; Geesey, G. G.; Gorby, Y. A.; Little, B. J. Geochim. Cosmochim. Acta 2003, 67, 4489.

(7) Kerisit, S.; Cooke, D. J.; Spagnoli, D.; Parker, S. C. J. Mater. Chem. 2005, 15, 1454.

(8) Dewan, S.; Yeganeh, M. S.; Borguet, E. J. Phys. Chem. Lett. 2013, 4, 1977.

(9) Hiemstra, T.; Venema, P.; Riemsdijk, W. H. V. J. Colloid Interface Sci. 1996, 184, 680 .

(10) Bickmore, B. R.; Rosso, K. M.; Tadanier, C. J.; Bylaska, E. J.; Doud, D. Geochim. Cosmochim. Acta 2006, 70, 4057.

(11) Eng, P. J.; Trainor, T. P.; Brown Jr., G. E.; Waychunas, G. A.; Newville, M.; Sutton, S. R.; Rivers, M. L. Science 2000, 288, 1029.

(12) Zhang, Z.; Fenter, P.; Cheng, L.; Sturchio, N. C.; Bedzyk, M. J.; Předota, M.; Bandura, A.; Kubicki, J. D.; Lvov, S. N.; Cummings, P. T.; Chialvo, A. A.; Ridley, M. K.; Bénézeth, P.; Anovitz, L.; Palmer, D. A.; Machesky, M. L.; Wesolowski, D. J. Langmuir 2004, 20, 4954.

(13) Catalano, J. G.; Zhang, Z.; Park, C.; Fenter, P.; Bedzyk, M. J. Geochim. Cosmochim. Acta 2007, 71, 1883.

(14) Spagnoli, D.; Gilbert, B.; Waychunas, G. A.; Banfield, J. F. Geochim. Cosmochim. Acta 2009, 73, 4023.
(15) Cheng, J.; Sprik, M. J. Chem. Theory Comput. 2010, 6, 880.

(16) von Rudorff, G. F.; Jakobsen, R.; Rosso, K. M.; Blumberger, J. J. Phys. Chem. Lett. 2016, 7, 1155.

(17) von Rudorff, G. F.; Jakobsen, R.; Rosso, K. M.; Blumberger, J. J. Phys.: Condens. Matter 2016, 28, 394001.

(18) Eftekhari-Bafrooei, A.; Borguet, E. J. Phys. Chem. Lett. 2011, 2, 1353.

(19) Fenter, P.; Sturchio, N. C. Prog. Surf. Sci. 2004, 77, 171.

(20) Tanwar, K. S.; Lo, C. S.; Eng, P. J.; Catalano, J. G.; Walko, D. A.; Brown Jr, G. E.; Waychunas, G. A.; Chaka, A. M.; Trainor, T. P. Surf. Sci. 2007, 601, 460 .

(21) Tanwar, K. S.; Catalano, J. G.; Petitto, S. C.; Ghose, S. K.; Eng, P. J.; Trainor, T. P. Surf. Sci. 2007, 601, L59.

(22) Catalano, J. G.; Fenter, P.; Park, C. Geochim. Cosmochim. Acta 2007, 71, 5313 .

(23) Kerisit, S.; Bylaska, E. J.; Massey, M. S.; McBriarty, M. E.; Ilton, E. S. Inorg. Chem. 2016, 55, 11736.

(24) Parkinson, G. S. Surf. Sci. Rep. 2016, 71, 272.

(25) Mackrodt, W. C.; Davey, R. J.; Black, S. N.; Docherty, R. J. Cryst. Growth 1987, 80, 441 .

(26) Guo, H.; Barnard, A. S. J. Mater. Chem. 2011, 21, 11566.

(27) Iordanova, N.; Dupuis, M.; Rosso, K. M. J. Chem. Phys. 2005, $122,144305$.

(28) Yanina, S. V.; Rosso, K. M. Science 2008, 320, 218.

(29) Chatman, S.; Zarzycki, P.; Preočanin, T.; Rosso, K. M. J. Colloid Interface Sci. 2013, 391, 125.

(30) Lo, C. S.; Tanwar, K. S.; Chaka, A. M.; Trainor, T. P. Phys. Rev. B 2007, 75, 075425 .

(31) Kerisit, S. Geochim. Cosmochim. Acta 2011, 75, 2043.

(32) McIntyre, N. S.; Zetaruk, D. G. Anal. Chem. 1977, 49, 1521.

(33) Wagner, C. D.; Davis, L. E.; Zeller, M. V.; Taylor, J. A.; Raymond, R. H.; Gale, L. H. Surf. Interface Anal. 1981, 3, 211.

(34) Yamamoto, S.; Kendelewicz, T.; Newberg, J. T.; Ketteler, G.; Starr, D. E.; Mysak, E. R.; Andersson, K. J.; Ogasawara, H.; Bluhm, H.; Salmeron, M.; Brown, G. E.; Nilsson, A. J. Phys. Chem. C 2010, 114, 2256.

(35) Henderson, M. A.; Joyce, S. A.; Rustad, J. R. Surf. Sci. 1998, 417, 66

(36) Robinson, I. K. Phys. Rev. B 1986, 33, 3830.

(37) Bjorck, M.; Andersson, G. J. Appl. Crystallogr. 2007, 40, 1174.

(38) Bjorck, M. J. Appl. Crystallogr. 2011, 44, 1198.

(39) Trainor, T. P.; Eng, P. J.; Robinson, I. K. J. Appl. Crystallogr.

2002, 35, 696 .

(40) Trainor, T. P.; Eng, P. J.; Brown Jr, G. E.; Robinson, I. K.; Santis, M. D. Surf. Sci. 2002, 496, 238.

(41) Krukau, A. V.; Vydrov, O. A.; Izmaylov, A. F.; Scuseria, G. E. J. Chem. Phys. 2006, 125, 224106.

(42) Pozun, Z. D.; Henkelman, G. J. Chem. Phys. 2011, 134, 224706.

(43) Grimme, S.; Ehrlich, S.; Goerigk, L. J. Comput. Chem. 2011, 32 , 1456.

(44) Guidon, M.; Hutter, J.; VandeVondele, J. J. Chem. Theory Comput. 2010, 6, 2348.

(45) VandeVondele, J.; Krack, M.; Mohamed, F.; Parrinello, M.; Chassaing, T.; Hutter, J. Comput. Phys. Commun. 2005, 167, 103. (46) Curtiss, L. A.; Halley, J. W.; Hautman, J.; Rahman, A. J. Chem. Phys. 1987, 86, 2319.

(47) Jorgensen, W. L.; Chandrasekhar, J.; Madura, J. D.; Impey, R. W.; Klein, M. L. J. Chem. Phys. 1983, 79, 926.

(48) Brown, I. D.; Altermatt, D. Acta Crystallographica Section B $\mathbf{1 9 8 5}, 41,244$.

(49) Bickmore, B. R. In Bond Valences; Brown, I. D., Poeppelmeier, K. R., Eds.; Springer Berlin Heidelberg: Berlin, Heidelberg, 2014, p 191. 


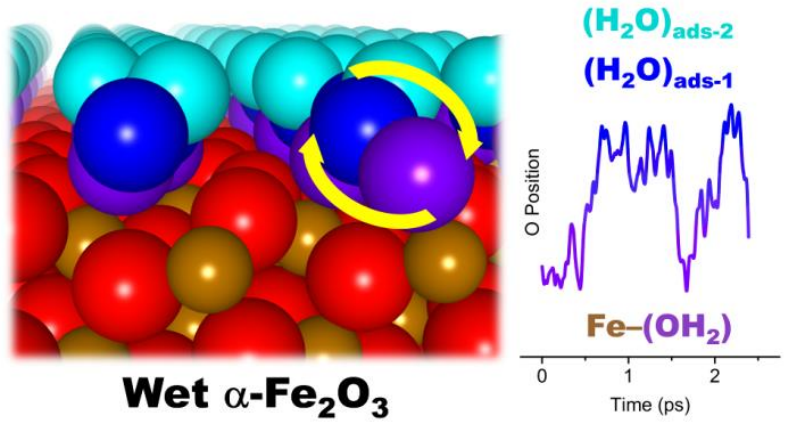

